\title{
UMD at SemEval-2018 Task 10: Can Word Embeddings Capture Discriminative Attributes?
}

\author{
Alexander Zhang and Marine Carpuat \\ Department of Computer Science \\ University of Maryland \\ College Park, MD 20742, USA \\ alexz@umd.edu, marine@cs.umd.edu
}

\begin{abstract}
We describe the University of Maryland's submission to SemEval-018 Task 10, "Capturing Discriminative Attributes": given word triples $\left(w_{1}, w_{2}, d\right)$, the goal is to determine whether $d$ is a discriminating attribute belonging to $w_{1}$ but not $w_{2}$. Our study aims to determine whether word embeddings can address this challenging task. Our submission casts this problem as supervised binary classification using only word embedding features. Using a gaussian SVM model trained only on validation data results in an F-score of $60 \%$. We also show that cosine similarity features are more effective, both in unsupervised systems (F-score of 65\%) and supervised systems (F-score of 67\%).
\end{abstract}

\section{Introduction}

SemEval-2018 Task 10 (Krebs et al., 2018) offers an opportunity to evaluate word embeddings on a challenging lexical semantics problem. Much prior work on word embeddings has focused on the well-established task of detecting semantic similarity (Mikolov et al., 2013a; Pennington et al., 2014; Baroni et al., 2014; Upadhyay et al., 2016). However, semantic similarity tasks alone cannot fully characterize the differences in meaning between words. For example, we would expect the word car to have high semantic similarity with truck and with vehicle in distributional vector spaces, while the relation between $c a r$ and truck differs from the relation between car and vehicle. In addition, popular datasets for similarity tasks are small, and similarity annotations are subjective with low inter-annotator agreement (Krebs and Paperno, 2016).
Task 10 focuses instead on determining semantic difference: given a word triple $\left(w_{1}, w_{2}, d\right)$, the task consists in predicting whether $d$ is a discriminating attribute applicable to $w_{1}$, but not to $w_{2}$. For instance, $\left(w_{1}=\right.$ apple, $w_{2}=$ banana, $d=$ red $)$ is a positive example as red is a typical attribute of apple, but not of banana.

This work asks to what extent word embeddings can address the challenging task of detecting discriminating attributes. On the one hand, word embeddings have proven useful for a wide range of NLP tasks, including semantic similarity (Mikolov et al., 2013a; Pennington et al., 2014; Baroni et al., 2014; Upadhyay et al., 2016) and detection of lexical semantic relations, either explicitly by detecting hypernymy, lexical entailment (Baroni et al., 2012; Roller et al., 2014; Turney and Mohammad, 2013), or implicitly using analogies (Mikolov et al., 2013b). On the other hand, detecting discriminating attributes requires making fine-grained meaning distinctions, and it is unclear to what extent they can be captured with opaque dense representations.

We start our study with unsupervised models. We propose a straightforward approach where predictions are based on a learned threshold for the cosine similarity difference between $\left(w_{1}, d\right)$ and $\left(w_{2}, d\right)$, representing words using Glove embeddings (Pennington et al., 2014). We use this unsupervised approach to evaluate the impact of word embedding dimensions on performance.

We then compare the best unsupervised configuration to supervised models, exploring the impact of different classifiers and training configurations. Using word embeddings as features, supervised models yield high F-scores on development data, on the final test set they perform worse than the unsupervised models. Our supervised submission yields an F-score of $60 \%$. In later experiments, we show that using cosine similarity as features 
is more effective than directly using word embeddings, reaching an F-score of $67 \%$.

\section{Task Data Overview}

\begin{tabular}{lrrrr}
\hline Dataset & Pos & Neg & Total & $d$ Vocab \\
\hline train & 6,591 & 11,191 & 17,782 & 1,292 \\
validation & 1,364 & 1,358 & 2,722 & 576 \\
\hline test & 1,047 & 1,293 & 2,340 & 577 \\
\hline
\end{tabular}

Table 1: Dataset statistics for the training and validation set: number of positive examples (Pos), number of negative examples (Neg), total number of examples (total), size of vocabulary for discriminant words $d$ ( $d$ Vocab)

For development purposes, we are provided with two datasets: a training set and a validation set, whose statistics are summarized in Table 1.

Word triples $\left(w_{1}, w_{2}, d\right)$ were selected using the feature norms set from McRae et al. (2005). Only visual discriminant features were considered for $d$, such as $i s_{-}$green. Positive triples $\left(w_{1}, w_{2}, d\right)$ were formed by selecting $w_{2}$ among the 100 nearest neighbors of $w_{1}$ such that a visual feature $d$ is attributable to $w_{1}$ but not $w_{2}$. Negative triples were formed by either selecting an attribute attributable to both words, or by randomly selecting a feature not attributable to either word.

The distribution of the training and validation sets differ: the validation and test sets are balanced, while only $37 \%$ of examples are positive in the training set. In addition, the validation and test sets were manually filtered to improve quality, so the training examples are more noisy. The data split was chosen to have minimal overlap between discriminant features.

\section{Unsupervised Systems}

All our models rely on Glove (Pennington et al., 2014), generic word embeddings models, pretrained on large corpora: the Wikipedia and English Gigaword newswire corpora. In addition to capturing semantic similarity with distances between words, Glove aims for vector differences to capture the meaning specified by the juxtaposition of two words, which is a good fit for our task.

Because the discriminant features are distinct between train, validation and test, our systems should be able to generalize to previously unseen discriminants. This makes approaches based on word embeddings attractive, as information about word identity is not directly encoded in our model.

\subsection{Baseline}

We first consider the baseline approach introduced by Krebs and Paperno (2016) to detect the positive examples, where $c s$ denotes the cosine similarity function:

$$
c s\left(\text { word }_{1}, d i s c\right)>c s\left(\text { word }_{2}, d i s c\right)
$$

\subsection{2-Step Unsupervised System}

We refine this baseline with a 2-step approach. Our intuition is that $d$ is a discriminant between $w_{1}$ and $w_{2}$ if the following two conditions hold simultaneously:

1. $w_{1}$ is more similar to $d$ than $w_{2}$ by more than a threshold $t_{\text {thresh }}$ :

$$
c s\left(w_{1}, d\right)-c s\left(w_{2}, d\right)>t_{\text {thresh }}
$$

2. $d$ is highly similar to $w_{1}$ :

$$
c s\left(w_{1}, d\right)>t_{\text {diverge }}
$$

The condition in Equation 2 aims at detecting negative examples that share the discriminant attribute, and the condition defined by Equation 3 targets negative examples that share a random discriminant. Thresholds $t_{\text {thresh }}$ and $t_{\text {diverge }}$ are hyper-parameters tuned on the train.txt.

\subsection{Results}

We evaluate unsupervised systems using word embeddings of varying dimensions on the validation set, and report averaged F-scores. As can be seen in Table 2, increasing the dimension of word embeddings improves performance for both systems, and the 2-step model consistently outperforms the baseline. The best performance is obtained by the 2-step model with 300-dimensional word embeddings. We therefore select these embeddings for further experiments.

\begin{tabular}{lrrrr}
\hline Vector Dim & 50 & 100 & 200 & 300 \\
\hline baseline & .5765 & .5965 & .6171 & .6183 \\
2-step model & .4034 & .6130 & .6266 & .6312 \\
\hline
\end{tabular}

Table 2: Averaged F-Score across GloVe Dimensions between our 2-step unsupervised system and the baseline from Krebs and Paperno (2016), for word vectors of size 50, 100, 200 and 300 . 


\section{Supervised Systems}

\subsection{Submitted System}

During system development, we consider a range of binary classifiers that operate on feature representations derived from word embeddings $\overrightarrow{w_{1}}, \overrightarrow{w_{2}}$ and $\vec{d}$. We describe the system used for submission which was selected based on 10-fold crossvalidation using the concatenation of the training and validation data.

Feature Representations We seek to capture the difference in meaning between $w_{1}$ and $w_{2}$ and its relation to the meaning of the discriminant word $d$. Given word embeddings for each of these words $\overrightarrow{w_{1}}, \overrightarrow{w_{2}}$ and $\vec{d}$, respectively, we therefore construct input features based on various embedding vector differences. We experimented with the concatenation of $\overrightarrow{w_{1}}, \overrightarrow{w_{2}}, \vec{d}, \overrightarrow{w_{1}}-\vec{d}$ and $\overrightarrow{w_{1}}-\vec{d}$. Based on cross-validation performance on training and validation data, we eventually settled on the concatenation of $\overrightarrow{w_{1}}-\vec{d}$ and $\overrightarrow{w_{1}}-\vec{d}$, which yields a compact representation of $2 D$ features, if $D$ is the embedding dimension.

Binary Classifier We consider a number of binary classification models found in scikit-learn: logarithmic regression (LR), decision tree (DT), naïve Bayes $(\mathrm{NB}), \mathrm{K}$ nearest neighbors $(\mathrm{KNN})$, and SVM with linear (SVM-L), and Gaussian (SVM-G) kernels. We compare linear combinations of word embeddings to the more complex combinations enabled by non-linear models.

Submission Our submission used the refined SVM-G trained on validation.txt. There were three input triplets for which one word was out of the vocabulary of the Glove embedding model: random predictions were used for these. This system achieved an F-Score of .6018. This is a substantial drop from the averaged crossvalidation F-scores obtained during development which reached F-scores of 0.9318 using crossvalidation on the validation and training sets together, and 0.9674 using cross-validation on the training set only. Using the released test dataset, truth.txt, we consider various experiments to understand the poor performance of the model.

\subsection{Analysis: Embedding Selection}

We first evaluate our hypothesis that word embeddings that perform well in the unsupervised setting would also, in general, perform well for classification. We vary embedding dimensions keeping the rest of the experimental set-up constant (train on validation.txt, evaluate on truth.txt).

Table 3 shows the performance of all supervised model configurations and of the 2-step unsupervised system. Increasing the word embedding dimensions improves the performance of the 2-step unsupervised system, as observed during the development phase (Section 3). However, the supervised classifier behaves differently: for several linear classifiers (e.g., LR, DT, SVM-L) the best performance is achieved with smaller word embeddings. For the non-linear SVM used for submission (SVM-G), varying the embedding dimensions has little impact on overall performance. The SVM-G classifier's performance is now on par with the linear classifiers, while it performed better on development data.

The best performance overall is achieved by the unsupervised model, and taken together, the supervised results suggest that the submitted system overfit the validation set, and was not able to generalize to make good predictions on test examples.

\subsection{Analysis: Feature Variants}

Motivated by the good performance of the unsupervised model based on cosine similarity, we consider four feature representations variants for the supervised classifiers, ${ }^{1}$ :

$$
\begin{aligned}
V 1 & =\left[c s\left(w_{1}, w_{2}\right), c s\left(w_{1}, w_{d}\right), c s\left(w_{2}, d\right)\right] \\
V 2 & =\left[V 1, w_{1}-w_{2}, w_{d}\right] \\
V 3 & =\left[V 1, w_{1}-w_{d}, w_{2}\right] \\
V 4 & =\left[V 1, c s\left(w_{1}-w_{2}, w_{d}\right), c s\left(w_{1}-w_{d}, w_{2}\right)\right]
\end{aligned}
$$

Variant V1 based only on cosine similarity between all pairs yields competitive F-scores from both the SVM-G and LR models (Table 4), and it competitive with the best-performing unsupervised model. We thus use it as a starting point for subsequent variants. Variants V2 and V3 encode the intuition that we expect $w_{1}-w_{2} \approx w_{d}$ and $w_{1}-w_{d} \approx w_{2}$ for positive examples, and therefore, it is possible that these input representations may perform better than the differencesonly model. In doing so, we also risk memorizing actual input words as $w_{d}$ and $w_{2}$ are encoded directly as features. These two variants performed worse than the cosine-only models, suggesting that cosine similarity captures semantic

\footnotetext{
${ }^{1}$ The KNN, SVM-L, and SVM-G used tuned hyperparameters.
} 


\begin{tabular}{|l|rrr|rrr|rrr|r|r|r|}
\hline \multirow{2}{*}{ Model } & \multicolumn{3}{|c|}{$\operatorname{dim}=50$} & \multicolumn{3}{|c|}{$\operatorname{dim}=100$} & \multicolumn{3}{c}{$\operatorname{dim}=200$} & \multicolumn{3}{c}{$\operatorname{dim}=300$} \\
& $\mathrm{~F}$ & $\mathrm{P}$ & $\mathrm{R}$ & $\mathrm{F}$ & $\mathrm{P}$ & $\mathrm{R}$ & $\mathrm{F}$ & $\mathrm{P}$ & $\mathrm{R}$ & $\mathrm{F}$ & $\mathrm{P}$ & $\mathrm{R}$ \\
\hline LR & .5742 & .5750 & .5741 & .5769 & .5788 & .5770 & .5739 & .5741 & .5738 & .5525 & .5525 & .5524 \\
$\mathrm{DT}$ & .5494 & .5498 & .5503 & .5356 & .5357 & .5359 & .5304 & .5311 & .5314 & .5283 & .5290 & .5293 \\
\hline NB & .5618 & .5674 & .5634 & .5873 & .5999 & .5903 & .5885 & .5904 & .5884 & .5908 & .5972 & .5918 \\
KNN & .5640 & .5746 & .5677 & .5677 & .5715 & .5720 & .5738 & .5737 & .5740 & .5537 & .5575 & .5579 \\
\hline SVM-L & .5769 & .5778 & .5768 & .5847 & .5904 & .5856 & .5781 & .5791 & .5779 & .5364 & .5372 & .5376 \\
SVM-G & .5901 & .5909 & .5919 & .6098 & .6099 & .6097 & .5924 & .5923 & .5924 & .5995 & .6002 & .5993 \\
\hline 2-step & .5937 & .5938 & .5947 & .6042 & .6041 & .6044 & .6278 & .6278 & .6290 & .6484 & .6481 & .6490 \\
\hline
\end{tabular}

Table 3: F-Score, Precision and Recall computed on truth.txt for the full range of supervised classification models across different embedding dimensions trained on validation.txt. The first 6 row are supervised systems, the last row shows the performance of the unsupervised 2-step system.

\begin{tabular}{lrrrr}
\hline Vector Dim. & 50 & 100 & 200 & 300 \\
\hline V1-LR & .6083 & .6076 & .6369 & .6526 \\
V1-KNN & .6045 & .6115 & .6335 & .6587 \\
V1-SVMG & .6039 & .6227 & .6479 & .6681 \\
\hline V2-LR & .6398 & .6475 & .6463 & .6490 \\
V2-KNN & .5376 & .5239 & .5334 & .5221 \\
V2-SVMG & .6304 & .6435 & .6592 & .6598 \\
\hline V3-LR & .6203 & .6108 & .6167 & .6193 \\
V3-KNN & .5356 & .5182 & .5116 & .5308 \\
V3-SVMG & .6099 & .6233 & .6269 & .6309 \\
\hline V4-LR & .6089 & .6072 & .6378 & .6525 \\
V4-KNN & .6088 & .6120 & .6402 & .6589 \\
V4-SVMG & .6102 & .6239 & .6451 & .6708 \\
\hline
\end{tabular}

Table 4: F-score for well-performing models of alternative input variant representations

difference better than the high-dimensional word vectors themselves. Also interestingly, the KNN model performed significantly worse in these two variants. The best result is achieved using V4, which augments $\mathrm{V} 1$ with cosine features that better capture word relations through embedding differences, with an averaged F-score of .6708 using the SVM-G classifier.

\subsection{Analysis: Cross-Validation Set-up}

We further explore why cross-validation scores differed greatly from the final test scores. We constructed initial cross-validation sets using sequential $10 \%$ cuts of the training set. This is inconsistent with the actual experimental setup, which had distinct sets of $d$, the discriminating attribute, between the training and test sets. We experiment segmenting the validation dataset so that each of the cross-validation sets had distinct discriminat- ing attributes. This yields only minor gains (Table 5), suggesting that overfitting to the identity of the discriminating attributes was not an issue.

\begin{tabular}{lrrrr}
\hline Vector Dim. & 50 & 100 & 200 & 300 \\
\hline V4-KNN & .6050 & .6172 & .6394 & .6574 \\
V4-SVML & .6109 & .6042 & .6301 & .6478 \\
V4-SVMG & .6104 & .624 & .6404 & .6716 \\
\hline
\end{tabular}

Table 5: F-score from well-formed crossvalidation sets

\section{Conclusion}

This study showed the limits of directly using word embeddings as features for the challenging task of capturing discriminative attributes between words. Supervised models based on raw embedding features are highly sensitive to the nature and distribution of training examples. Our Gaussian Kernel SVM overfit the training set and performed worse than unsupervised models that threshold cosine similarity scores on the official evaluation data. Based on this finding, we explore the use of cosine similarity scores as features for supervised classifiers, to capture similarity between word pairs, and between words and word relations as represented by embedding differences. These features turn out to be more useful than directly using the word embedding themselves, yielding our best performing system (Fscore of $67 \%$ ).

While these results are encouraging, it remains to be seen how to best design models and features that capture nuanced meaning differences, for instance by leveraging metrics complementary to cosine and resources complementary to distributional embeddings. 


\section{References}

Marco Baroni, Raffaella Bernardi, Ngoc-Quynh Do, and Chung-chieh Shan. 2012. Entailment above the word level in distributional semantics. In Proceedings of EACL 2012, pages 23-32.

Marco Baroni, Georgiana Dinu, and Germán Kruszewski. 2014. Don't count, predict! A systematic comparison of context-counting vs. context-predicting semantic vectors. In $A C L$ (1), pages 238-247.

Alicia Krebs, Alessandro Lenci, and Denis Paperno. 2018. Semeval-2018 task 10: Capturing discriminative attributes. In Proceedings of SemEval-2018. International Workshop on Semantic Evaluation.

Alicia Krebs and Denis Paperno. 2016. Capturing discriminative attributes in a distributional space: Task proposal. In RepEval@ACL.

Ken McRae, George S. Cree, Mark S. Seidenberg, and Chris Mcnorgan. 2005. Semantic feature production norms for a large set of living and nonliving things. Behavior Research Methods, 37(4):547-559.

Tomas Mikolov, Kai Chen, Greg Corrado, and Jeffrey Dean. 2013a. Efficient estimation of word representations in vector space. In arXiv Preprint.

Tomas Mikolov, Wen-tau Yih, and Geoffrey Zweig. 2013b. Linguistic Regularities in Continuous Space Word Representations. In HLT-NAACL, volume 13, pages 746-751.

Jeffrey Pennington, Richard Socher, and Christopher D. Manning. 2014. GloVe: Global Vectors for Word Representation. In EMNLP 2014, pages 1532-1543.

Stephen Roller, Katrin Erk, and Gemma Boleda. 2014. Inclusive yet Selective: Supervised Distributional Hypernymy Detection. Proceedings of COLING 2014, pages 1025-1036.

Peter Turney and Saif Mohammad. 2013. Experiments with three approaches to recognizing lexical entailment. Natural Language Engineering, 1(1):1-42.

Shyam Upadhyay, Manaal Faruqui, Chris Dyer, and Dan Roth. 2016. Cross-lingual Models of Word Embeddings: An Empirical Comparison. In Proceedings of $A C L$, Berlin, Germany. 